\title{
Keck Interferometer Nuller Science Highlights
}

\author{
Bertrand Mennesson*a, Rafael Millan-Gabet ${ }^{\mathrm{b}}$, M.M. Colavita $^{\mathrm{a}}$, E. Serabyn ${ }^{\mathrm{a}}$, P. Hinz ${ }^{\mathrm{c}}$, M. Kuchner ${ }^{\mathrm{d}}$, \\ W. Liu ${ }^{\mathrm{e}}$, R. Barry ${ }^{\mathrm{d}}$, C. Stark ${ }^{\mathrm{f}}$, S. Ragland ${ }^{\mathrm{g}}$, J. Woillez ${ }^{\mathrm{g}}$, W. Traub ${ }^{\mathrm{a}}$, O. Absil ${ }^{\mathrm{h}}$, D. Defrere ${ }^{\mathrm{i}}$, J.C. \\ Augereau ${ }^{\mathrm{j}}, \mathrm{J}$. Lebreton $^{\mathrm{j}}$

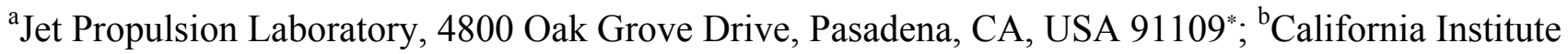 \\ of Technology, NASA Exoplanet Science Institute, Pasadena, CA 91125, USA; 'Steward \\ Observatory, The University of Arizona, 933 N. Cherry Ave., Tucson, AZ 85721, USA; ${ }^{\mathrm{d}} \mathrm{NASA}$ \\ Goddard Space Flight Center, Exoplanets and Stellar Astrophysics Laboratory, Code 667, Greenbelt, \\ MD 20771, USA; ' Infrared Processing and Analysis Center, California Institute of Technology, MC \\ 100-22, 770 South Wilson Avenue, Pasadena, CA 91125, USA; ${ }^{\mathrm{f}}$ Carnegie Institution for Science. \\ Department of Terrestrial Magnetism. 5241 Broad Branch Road. Washington, D.C. 20015; ${ }^{\mathrm{g}}$ Keck \\ Observatory, 65-1120 Mamalahoa Hwy, Kamuela, HI 96743, USA; ${ }^{\mathrm{h}}$ Dept. d'Astrophysique, \\ Géophysique \& Océanographie, Université de Liège, 17 Allée du Six Août, 4000, Liège, Belgium; \\ ${ }^{\mathrm{i}}$ Max Planck Institut für Radioastronomie, Auf den Hügel 69, 53121, Bonn, Germany; ${ }^{\mathrm{j}}$ Institut de \\ Planétologie et d'Astrophysique de Grenoble (IPAG) UMR 5274, 38041, Grenoble, France
}

\begin{abstract}
We report here on some of the major astronomical observations obtained by the Keck Interferometer Nuller (KIN), the high dynamic range instrument recombining the Keck Telescopes at wavelengths of 8 to 13 microns. A few science targets were observed during the commissioning phase (2004-2007). These early observations aimed at demonstrating the KIN's ability to spatially resolve and characterize circumstellar dust emission around a variety of targets, ranging from evolved stars to young debris disks. Science operations started then in 2008 with the more demanding KIN exozodi key science programs, augmented by observations of YSOs and hot debris disks between 2009 and 2011. The last KIN observations were gathered in $2011 \mathrm{~B}$, and the interpretation of some of the results depicted here is still preliminary (exo-zodi survey) or pending (complicated behavior observed in YSOs). We discuss in particular the initial results of the KIN's exo-zodi observations, which targeted a total of 40 nearby main sequence single stars. We look for trends in this sample, searching for possible correlations between the measured KIN excesses and basic stellar properties such as spectral type or the presence of dust inferred from separate observations.
\end{abstract}

Keywords: Nulling- Exozodiacal Light - Infrared - Young Stars - Symbiotic Stars - Main Sequence Stars

\section{INTRODUCTION}

The 4-beam nulling architecture of the KIN has been detailed in a number of previous publications (e.g. Serabyn et al. $2012^{1}$ ), and will not be discussed here. The main performance driver for the KIN is dynamic range, as required to detect faint exo-zodi structures around nearby main sequence stars, and the instrument was correspondingly designed to provide very accurate null (or equivalently visibility) measurements in the mid-infrared. By the end of the commissioning phase (2004-2007), the measured null accuracy was established to be $\sim 0.2 \%(1 \sigma)$ per night for bright targets and for all wavelengths ranging from 8 to 11 microns (Colavita et al. 2009²). The KIN's other key instrumental parameters are (i) its spatial resolution (defined as the nuller's $50 \%$ transmission point) which goes from 10 mas at 8 microns to about 15 mas at 13 microns, (ii) its spectral resolution of about 20:1 (providing 10 spectral channels across the $\mathrm{N}$ band), (iii) its sensitivity of 1.5 Jy at 10 microns, and finally (iv) its effective field of view, which is restricted to about 0.4 " x 0.1 " (in

* Bertrand.Mennesson@jpl.nasa.gov; phone 1818 354-0494

Optical and Infrared Interferometry III, edited by Françoise Delplancke, Jayadev K. Rajagopal, Fabien Malbet, Proc. of SPIE Vol. 8445, 844507 - (c) 2012 SPIE · CCC code: 0277-786/12/\$18 · doi: 10.1117/12.926548 
radius) around the central nulled target. This limited field of view means that only the very immediate circumstellar environment can be characterized by the nuller, e.g. regions located within a few AU for representative targets of the exo-zodi survey programs.

We follow hereafter a chronological path, starting with the observations conducted during the KIN commissioning (2004-2007). This phase aimed at demonstrating the potential of the instrument by observing increasingly challenging targets: evolved stars with previously known (but yet unresolved) dust shells, evolved stars with suspected dust, and finally a suspected young debris disk. We then report on some of the science observations which followed, concentrating on null observations of various types of YSOs (PIs: Ragland and Millan-Gabet), and on the characterization of exozodiacal light levels around nearby main sequence stars (3 key science programs conducted in 2008-2009 with respective PIs: Serabyn, Hinz and Kuchner, and one hot disk follow-up program conducted in 2010-2011, PI: Mennesson).

For centrally peaked sources, the observed null depth $\mathrm{N}$ can be simply related to the standard source complex visibility $\mathrm{V}$ at the Keck-Keck $\sim 85 \mathrm{~m}$ baseline by $\mathrm{N}=(1-|\mathrm{V}|) /(1+|\mathrm{V}|)$. For a perfect nulling instrument observing a point-like source, a zero null depth is then expected. In practice however, a non-zero instrumental null depth is generally measured. As in standard interferometric observations, this instrumental null depth (or visibility reduction) is estimated through measurements of calibrators with well-known diameters, and a calibrated null or visibility measurement is derived. As they are equivalent observables, we will either use visibility or null quantities to represent the KIN measurements in what follows. The next step in the data analysis process - specially relevant in the case where small excesses are searched-, is to subtract the null leakage resulting from partially resolving the central star, which yields the main KIN observable and quantity of interest, called "excess null" or "excess leakage" hereafter (section 4).

\section{COMMISSIONING OBSERVATIONS}

While the main goal of the KIN commissioning was to validate all technical functionalities, we also carried some scientific observations during that phase in order to assess the instrument readiness for science operations. We started with "easy" targets with previously known bright circumstellar dust emission, and followed with observations of stars with suspected extra-emission at lower levels.

\subsection{Evolved stars with known dust: RS CrB and TU Tau}

One of the first targets for which N-band fringes were obtained is RS CrB (Fig. 1), a semi regular variable giant with strong silicate emission features previously detected by IRAS, and classified as "SE6" in Sloan \& Price $1998^{3}$ silicate emission sequence. The KIN observations allowed a non-ambiguous determination of the dust shell spatial scale and relative flux contribution. Using a simple spherically symmetric model, in which a geometrically thin shell surrounds the stellar photosphere, we found that $\sim 30 \%$ to $\sim 70 \%$ of the overall mid-infrared flux -depending on the wavelengthoriginated from 7-8 stellar radii (Mennesson et al. $2005^{4}$ ). We also found that the shell opacity profile derived from the observations showed a broad peak around $11 \mu \mathrm{m}(\tau \sim=0.06)$, characteristic of Mg-rich silicate dust particles, and consistent with the observed IRAS spectrum. Interestingly, these are very similar findings to those of MIDI on the Mira star RR Sco (Ohnaka et al. 2005 $)$ which revealed an optically dust shell with an inner radius of 7-8 $\mathrm{R}^{*}$ as well, contributing to most of the excess observed longward of 10 microns. In order to further check the KIN's ability to disentangle between various spectral signatures and circumstellar symmetry, we also observed the symbiotic C-rich evolved star TU Tau, revealing the expected emission feature of $\mathrm{SiC}$, and some possible asymmetry in the direction of the hot (A type) companion (Woillez et al. unpublished). 

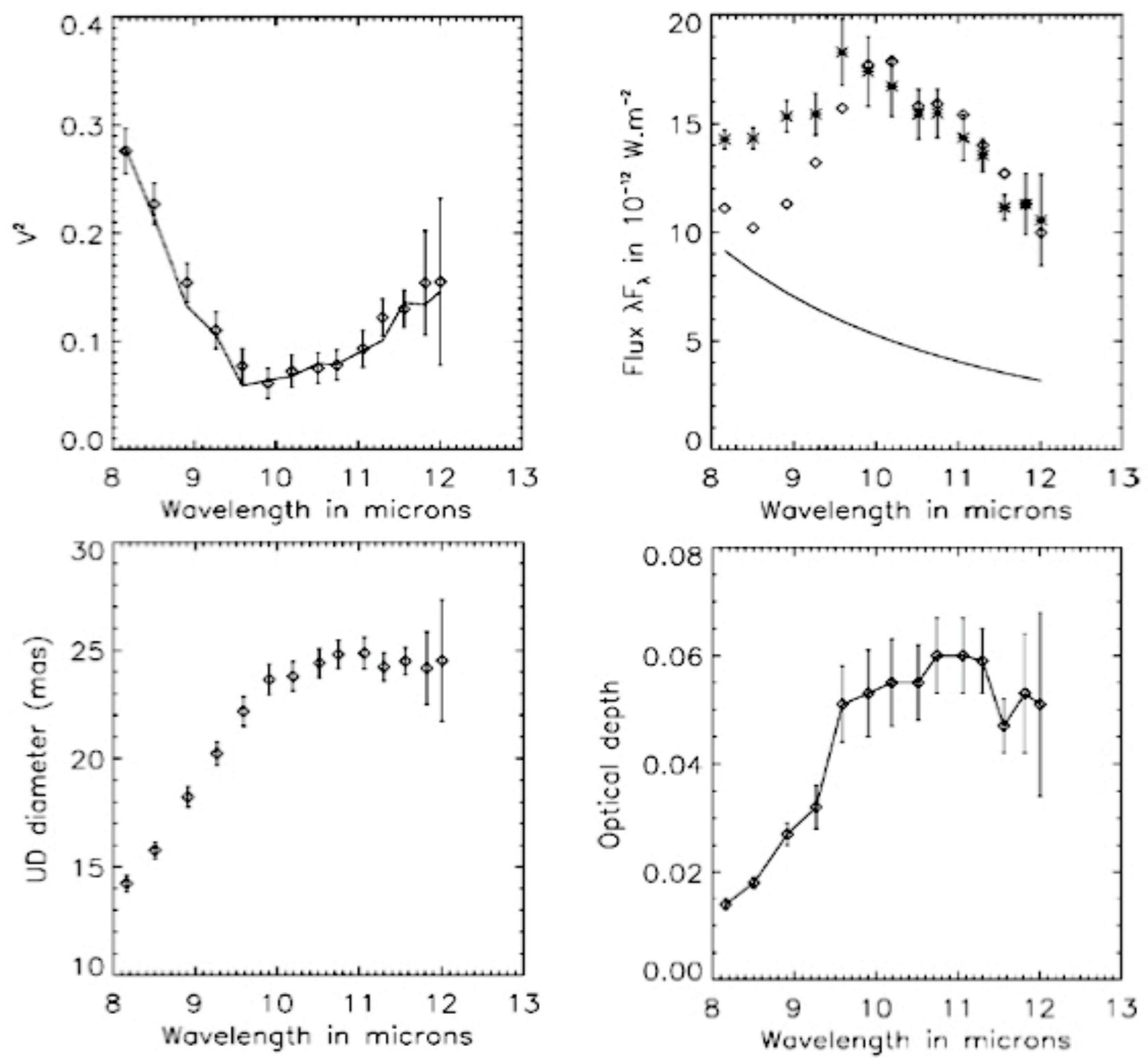

Figure 1: KIN observations of the semi-regular variable giant RS CrB (from Mennesson et al. 2005 ${ }^{4}$ ). Upper left panel: Diamonds and error bars $(1 \sigma)$ : observed squared visibility vs wavelength. Solid curve: Best model fit using a 2component model with a central photosphere ( 3.8 mas in diameter) and a surrounding dust shell ( 28 mas in diameter, at a temperature of $\sim 1160 \mathrm{~K}$ ). Lower left panel: stellar diameter derived from the observed visibilities, assuming a simple non-physical uniform disk model. Upper right panel: Crosses and error bars: Observed spectrum using the individual Keck telescopes. Diamonds: IRAS LRS spectrum converted to KIN's spectral resolution. Solid curve: Naked star spectrum, for the best fit photospheric diameter. Lower right panel: the observed variation of visibility vs wavelength can be reproduced by varying the opacity of a single dust layer across the $\mathrm{N}$ band. The derived shell opacity profile shows a broad peak around $11 \mu \mathrm{m}(\tau \sim 0.06)$, characteristic of $\mathrm{Mg}$-rich silicate dust particles.

\subsection{Evolved stars with suspected dust: X Gem and RS Oph}

- The Mira star X Gem is known to have periodic ( 260 days) flux variations of about 5 magnitudes in the visible, pointing to a significant mass loss rate and suggesting that molecules and/or dust are present in abundance in the immediate stellar environment. Although suspected to host dust, no excess had been previously detected by 
spectroscopic mid-infrared observations of X Gem(fig 2, left), making it an ideal target to validate the KIN's accuracy and ability to discover dust. Indeed, our $2005 \mathrm{KIN}$ measurements (fig 2, right) clearly resolved a broad emission feature within a few stellar radii of $\mathrm{X}$ Gem, contributing $\sim 10 \%$ of the total flux across the $\mathrm{N}$ band.
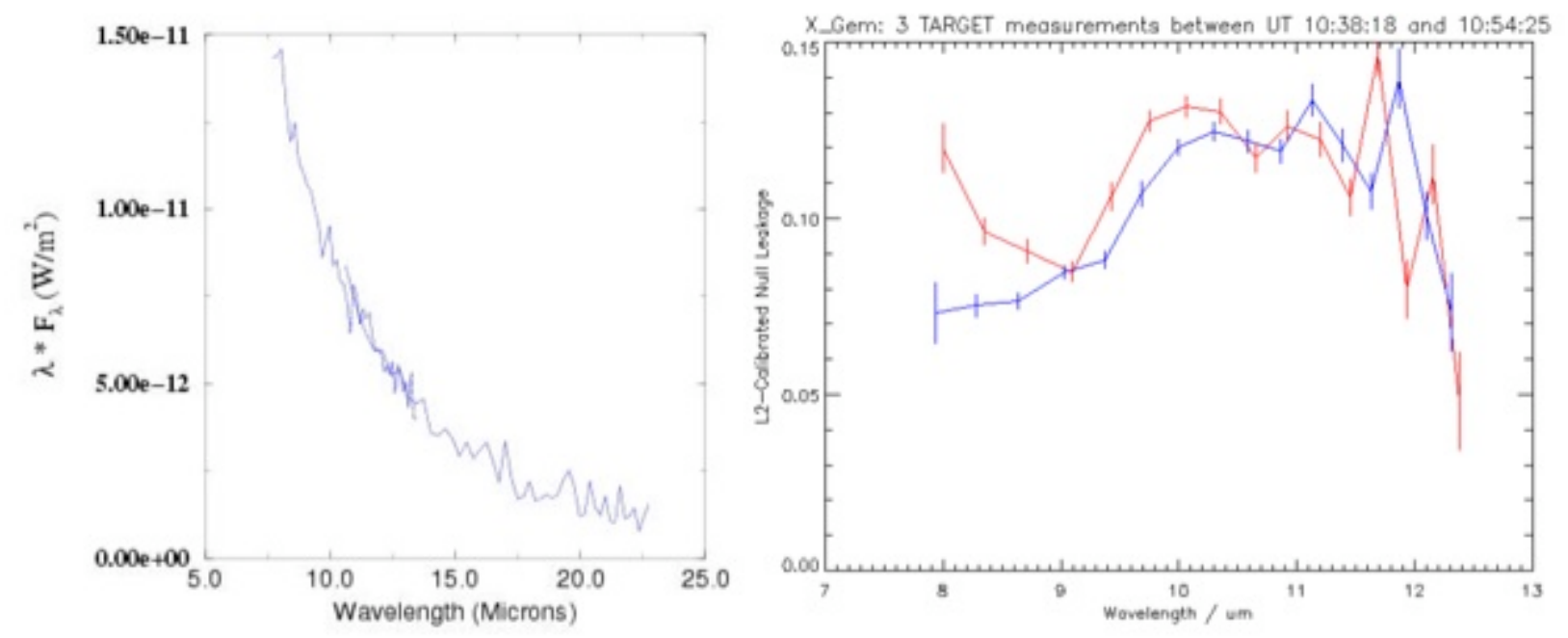

Figure 2: Left: IRAS LRS spectrum of $\mathrm{X}$ Gem, showing no obvious excess emission in the mid-infrared. Right: Calibrated nulls measured with the KIN as a function of wavelength. The red and blue curves correspond to two different camera ports and null beam combiner outputs. In both cases, a clear $\sim 5$ to $15 \%$ excess is detected over the signal expected from the photosphere alone (contributing about $2 \%$ null at 10 microns), and is probably due to resolved silicate emission in the CS environment.

- RS Oph is a recurrent nova (period of about 20 years, component separation of $\sim 1.7 \mathrm{AU}$ ), which happened to go through an outburst during a KIN commissioning run. We observed it on February 16 2006, about 3.8 days after its outburst (Barry et al. 2008 ${ }^{6}$ ). Figure 3 compares 2 different spectra recorded with the KIN: one at constructive interference (dominated by the central erupting white dwarf) and one at null (i.e. more sensitive to circumstellar emission). As expected, the "constructive" (upper panel) spectrum shows enhanced emission from neutral atomic $\mathrm{H}$ and metals $(\mathrm{Si}, \mathrm{Ca}, \mathrm{Mg})$, as expected from ejection of accumulated $\mathrm{H}$ layers and white dwarf surface material. As for the spectrum obtained when nulling the central white dwarf (lower panel), it shows enhanced emission from hot silicate dust, originating further away than the region reached by the blast wave. This result came as a surprise: it proved that dust was already there prior to the outburst, and not formed because of the compression effect of the blast on the red giant wind. A possible explanation for the presence of this large amount of dust (cleared every 20 years or so) is that it is instead produced by an increase in density in the plane of the orbit of the two stars, with its spatial distribution following an Archemidian spiral pattern, periodically destroyed after each blasting event. 


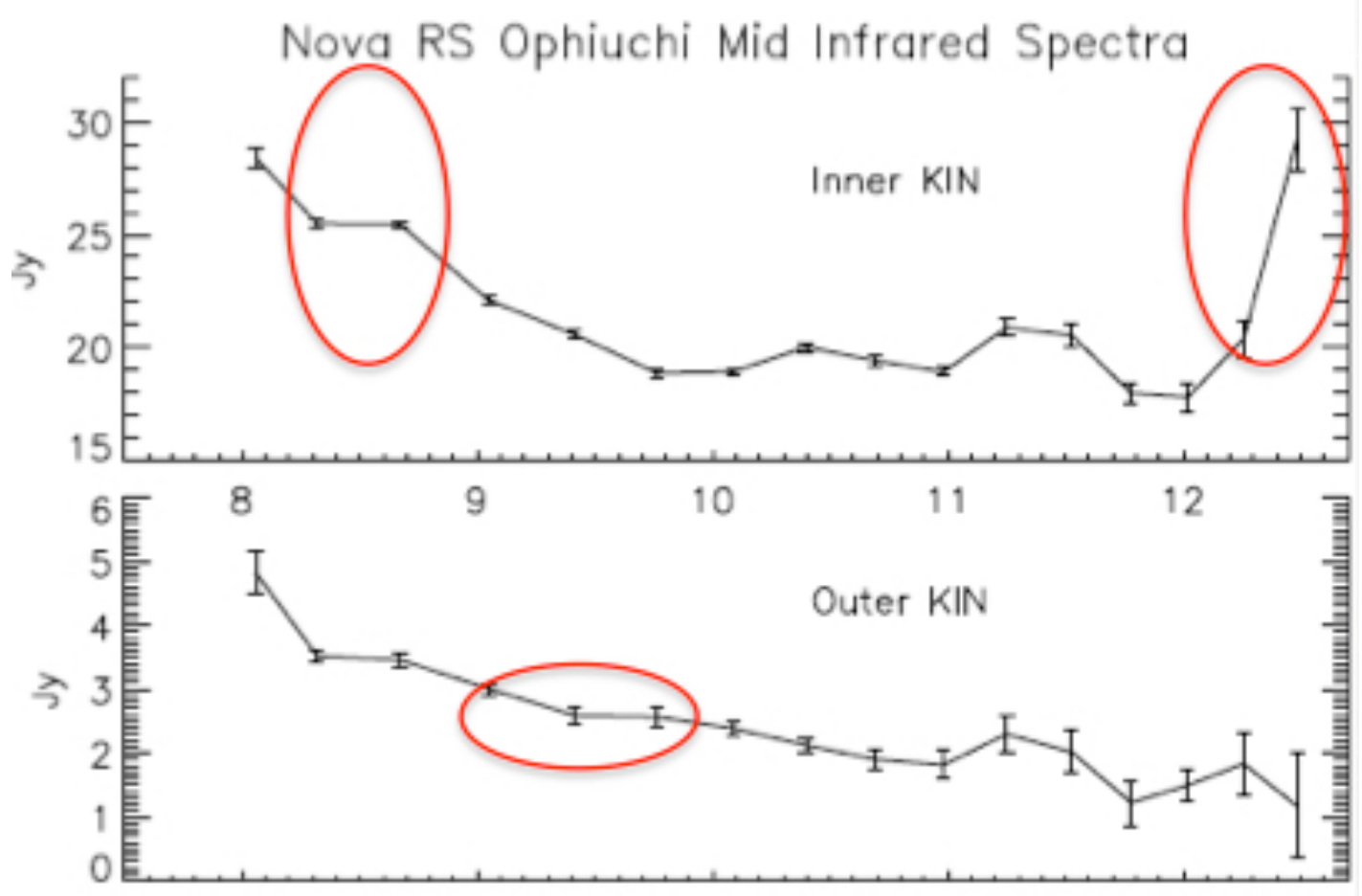

Figure 3. Comparison of spectra of the recurrent nova RS Oph measured with the KIN at peak (upper panel) and at null (lower panel). Regions highlighted in red show the largest disagreement. In particular, the lower panel measurements, more sensitive to the white dwarf circumstellar environment, show an enhanced emission likely due to hot silicate dust (Barry et al. $2008^{6}$ ). See text for details.

\subsection{Young Debris Disk 51 Oph}

In April 2007, we observed the 51 Ophiuchi circumstellar disk. Possibly a pre- main sequence star with a Herbig Be disk or a young debris disk with gas likely oriented edge-on but never imaged directly, this object was an ideal commissioning target for the KIN. The KIN data were modeled simultaneously with VLTI-MIDI visibility data and a Spitzer IRS spectrum using a variety of optically-thin dust cloud models and an edge-on optically thick disk model (Stark et al. $2009^{7}$ ). It was found that single-component optically-thin disk models and optically-thick disk models were inadequate to reproduce the observations, but that an optically-thin two-component disk model could reproduce all of the major spectral and interferometric features. The preferred disk model consisted of an inner disk of blackbody grains extending to $\sim 4 \mathrm{AU}$ and an outer disk of small silicate grains extending out to $\sim 1200 \mathrm{AU}$. This model is consistent with an inner "birth" disk of continually colliding parent bodies producing an extended envelope of ejected small grains. This picture resembles the disks around Vega, AU Microscopii, and $\beta$ Pictoris, supporting the idea that 51 Ophiuchius may be a $\beta$ Pictoris analog.

\section{SCIENCE OBSERVATIONS: YOUNG STELLAR OBJECTS}

Disks around YSOs are more extended and optically thick than debris disks around main sequence stars, making them fairly easy targets for the KIN, whose high accuracy visibility measurements is unique in the mid-infrared. One such observing program was led by Rafael Millan-Gabet with the goal of providing new information on YSOs mid-infrared spatial scales, constrain and test state of the art models for a variety of objects (7 Herbig AeBe stars, 3 T Tauris and 2 FU 
Oris). Observations were also coordinated with contemporaneous SED measurements at IRTF led by M. Sitko. Fig 4 presents the calibrated null depth measured as a function of wavelength for a subset of the YSOs sample, showing a large diversity of observed spectral signatures, even for objects of the same type (e.g. the Herbigs AB Aur, MWC275 and MWC 480). The interpretation and full modeling of these data is under progress (Millan-Gabet et al. in prep).
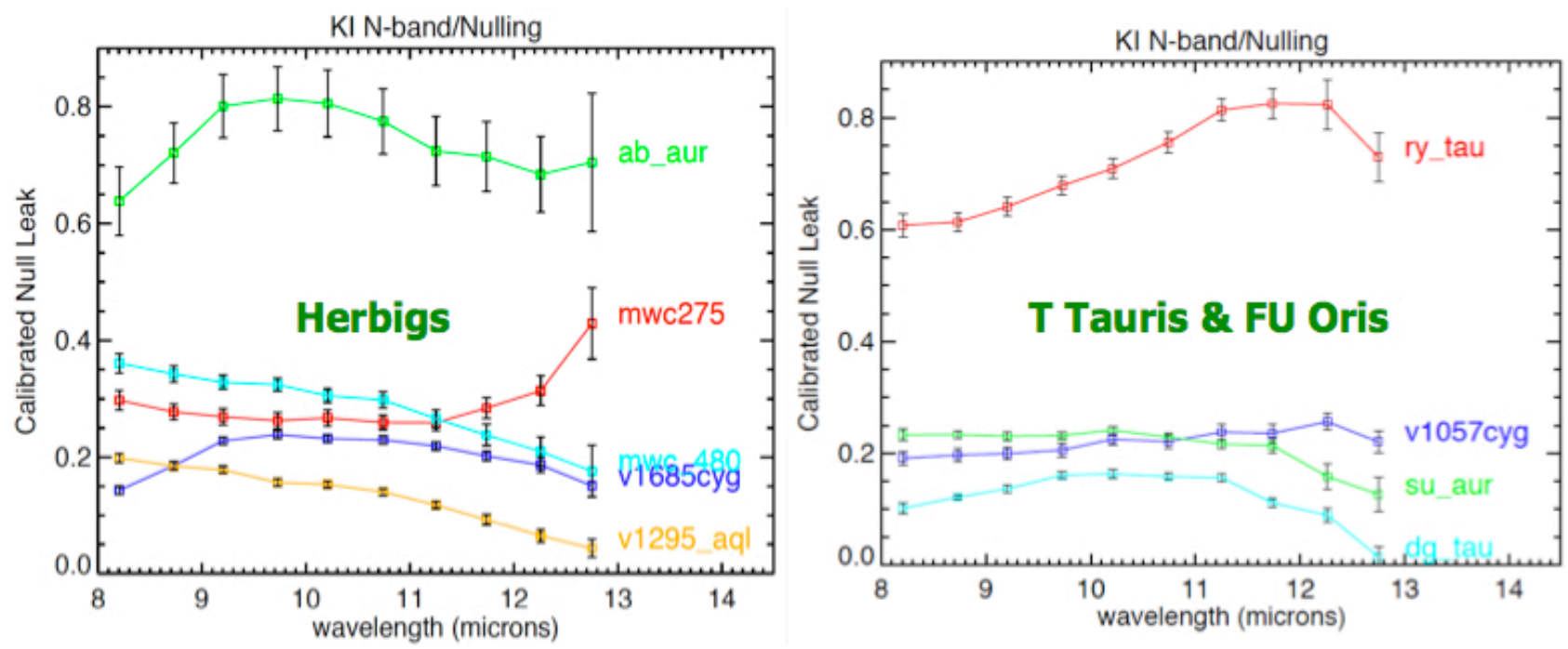

Figure 4. KIN observations of different types of YSOs illustrating the diversity of observed spectral null signatures (Millan-Gabet et al., in prep.)

Parallel observations of YSO disks were conducted at KI by L. Hillenbrand and S. Ragland. This work included the first KIN observations of a YSO, probing the circumstellar environment of the Herbig AeBe star MWC 325 (Ragland et al. $2012^{8}$ ). These KIN mid-infrared measurements were completed by regular KI visibility measurements at K and L bands, then encompassing a factor of five in spectral range. Fitting the observations with geometric models such as a uniform disk or a Gaussian disk show that the apparent size increases monotonically with wavelength in the 2-12 $\mu \mathrm{m}$ wavelength region, confirming the widely held assumption based on radiative transfer models, now with spatially resolved measurements over broad wavelength range, that disks are extended with a temperature gradient. The effective size is a factor of about 1.3 and 2 larger in the L-band and N-band, respectively, compared to that in the K-band. The existing interferometric measurements and the spectral energy distribution can be reproduced by a flat disk or a weakly shadowed nearly flat-disk model, with only slight flaring in the outer regions of the disk, consisting of representative "sub-micron" $(0.1 \mu \mathrm{m})$ and "micron" $(2 \mu \mathrm{m})$ grains of a 50:50 ratio of silicate and graphite. This is in marked contrast with the disks previously found in other Herbig Ae/Be stars, suggesting a wide variety in the disk properties among Herbig Ae/Be stars, as also evident from the initial results of the YSOs survey led by Millan-Gabet.

\section{SCIENCE OBSERVATIONS: KIN EXOZODI SURVEYS}

There are several reasons for studying the intensity and spatial distribution of debris disk components located in the inner few AUs of main sequence stars, i.e exozodiacal structures analogous to the solar zodiacal cloud and located where the planets are formed. The inner ( 0.1 to $\sim 10 \mathrm{AU})$ brightness distribution of exozodiacal dust in debris disks -which only long baseline interferometry can probe around a significant number of stars - reflects present dust sources (comets, asteroids), as well as sinks (Poynting-Robertson drag, radiation pressure), and perturbations (collisions, evaporation, planets), revealing some of the system's current dynamical state and formation history. Apart from providing such key information, exozodiacal disks also represent important noise and confusion sources for the direct imaging and spectroscopy of planets around other stars, particularly in the case of faint Earth-like exoplanets. A better knowledge of exo-zodi brightness level and morphology (both per individual star and in a statistical sense) plays then a prominent 
role for the design of future space missions aiming at the characterization of Earth-like exoplanets, whether using visible coronagraphy or mid-infrared interferometry. This issue was recognized early on (e.g. Beichman \& Velusamy $1997^{9}$, Mennesson \& Mariotti $1997^{10}$ ). The morphology and adverse effects of exozodical structures have also been studied in greater detail in recent papers (e.g. Defrere et al. $2010^{11}$, Stark and Cahoy 201122).

A total of 48 nearby main sequence stars have been surveyed by the KIN between 2008 and 2011: 23 stars with no previously known dust (most of them in the 2008-2009 key science survey led by E. Serabyn), 19 with cold dust previously detected in the mid or far IR (2008-2009 key science surveys led by P. Hinz and M. Kuchner), and 6 stars with hot dust emission suspected from previous near-infrared interferometric observations (2010-2011 survey led by B. Mennesson).

\subsection{Analysis of KIN observations of stars with a priori no dust}

The results of the detailed analysis of this sub-sample have been published recently (Millan-Gabet et al. $2011^{13}$ ). We basically found that only one star in this sample showed dust resolved by the KIN at a marginal level (Altair). Using a model of our own Solar System's zodiacal cloud, scaled to the luminosity of each target star, we estimated the equivalent number of target zodis needed to match our observations. The 22 non-detection have an ensemble weighted average consistent with zero, with an average individual uncertainty of 160 zodis. These measurements represent the best limits to date on exozodi levels for a sample of nearby main sequence stars. They are typically a factor of 2 better than the SPITZER limits, with the additional advantage that the KIN measurements resolve the dust emitting region, do not make any assumption on the stellar spectrum (e.g. its strictly photometric nature up to a certain wavelength). A statistical analysis of this population of 23 stars not previously known to contain circumstellar dust suggests that, if they are representative of a single class with respect to the level of exozodi brightness, the mean exozodi level for the class is $<150$ zodis ( $3 \sigma$ upper limit, corresponding to $99 \%$ confidence under the additional assumption that the measurement errors are Gaussian). Some stars in this sample show particularly "clean" non-detections (as illustrated in figure 5). They will feed into the LBTI nuller exo-zodi key science survey, in the category of "a priori non-dusty" nearby main sequence stars.

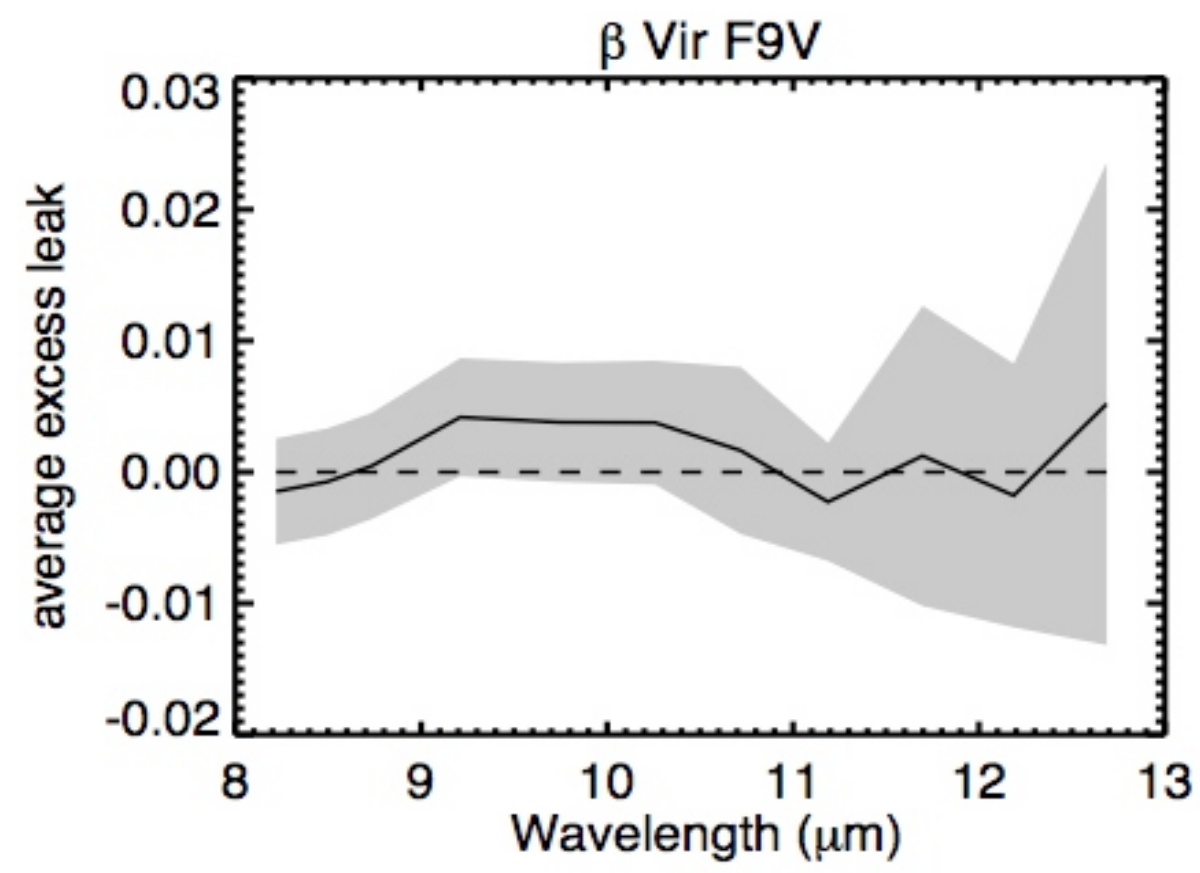

Figure 5: Example of a KIN non-detection. Solid curve: excess leak as a function of wavelength, measured for $\beta$ Vir (average and error bars are based on 7 null sequences, recorded on Feb. 17 \& 18, 2008). Shaded grey area: bracketing +/$1 \sigma$ confidence interval. 


\subsection{Analysis of KIN observations of stars with suspected dust (work in progress)}

A total of 25 stars with excesses previously detected at various infrared wavelengths (K-band: CHARA/FLUOR or VLTI/VINCI, far/mid IR: IRAS or SPITZER) were observed. Four of them were spectroscopic binaries and were ignored in the initial analysis summarized here (assessing tiny excesses is in this case quite problematic unless the orbital parameters and flux ratios are perfectly known). Of the remaining 21 stars, 5 show a mid IR excess detected by KIN at above $3 \sigma$ significance level. 6 more stars show a marginal excess detection at the $>2 \sigma$ level. In particular, only 3 of the stars with excesses detected at 2 microns show an excess with the KIN, and all 3 at small levels $(<2 \%)$. This indicates that in all cases, the phenomenon responsible for the near infrared excess is not contributing much flux at 10 microns. This either points to some unexpected chromospheric effects, or more likely to very "non-solar" dust populations, with very small hot grains piling up close to sublimation radius. Since such small grains should be blown out and expelled rapidly from the inner system, high replenishment rates or mechanisms are needed to trap small dust over long periods. At this stage, this mature stars "hot disk" phenomenon is still unexplained and its origin remains unclear.

Of particular interest among the stars showing a KIN excess is the case of Fomalhaut, which previously showed evidence for warm dust inside of 20 AU with Spitzer IRS (17.5 $\mu \mathrm{m}$ to $34 \mu \mathrm{m}$ data), and possibly even hotter dust grains located inside of 5 AU inferred from near infrared VLTI data (Absil et al. 2009). The mean KIN observed excess between 8 and 11 microns is $\sim 0.35 \%$, while that measured by the VLTI at K-band is close to $1 \%$. Assuming that these two measurements, taken at different epochs, characterize the same circumstellar environment, and conducting a detailed radiative transfer simulation, (Mennesson et al. in prep.), these observations point to a population of hot dust grains piling up close to the sublimation radius. Additionally, these grains must be small enough $(<1 \mu \mathrm{m})$ to strongly emit in the near infrared without causing a large excess at 10 microns. An other interesting feature of the KIN detected excess is that it increases quite sharply with wavelength between 8 and 13 microns (Fig. 6), suggesting a second population of larger ( $\sim$ microns) grains located at $0.5-1 \mathrm{AU}$ and further.

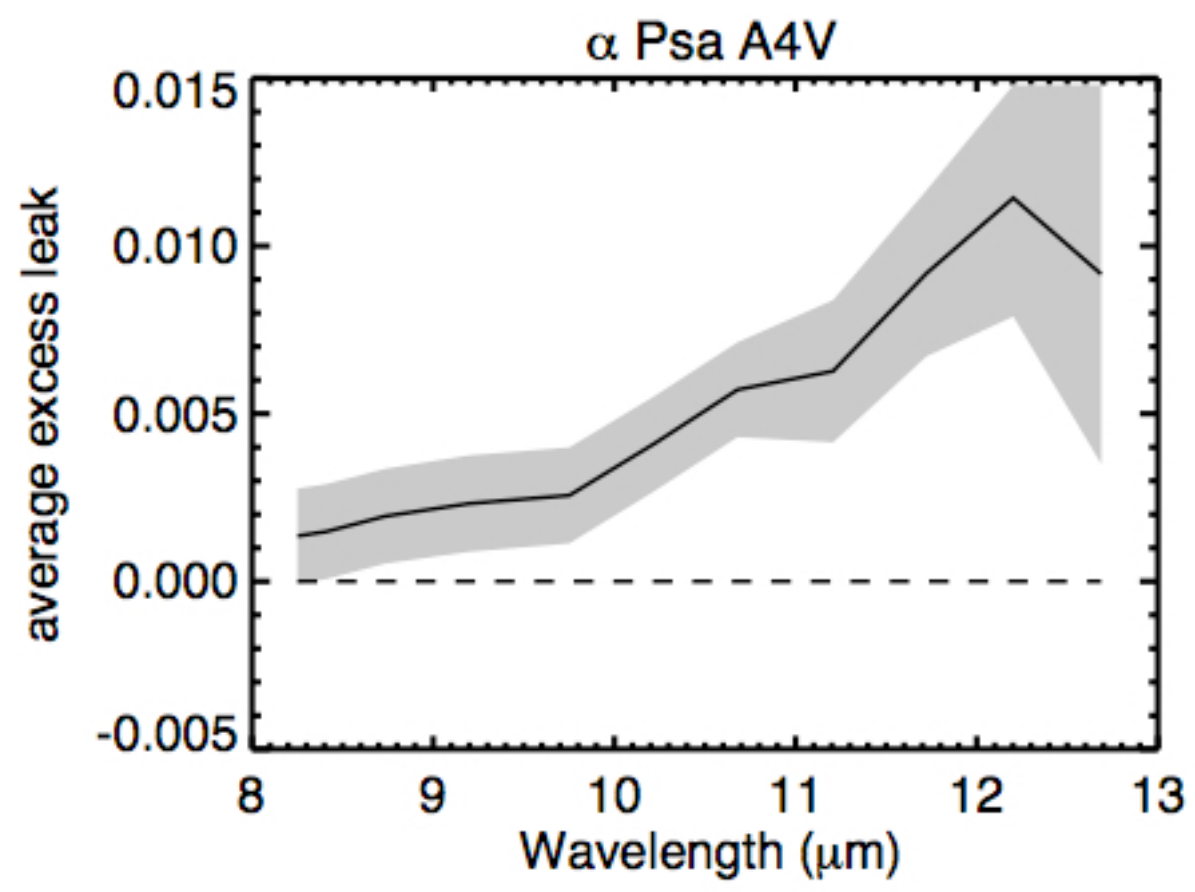

Figure 6: Example of a KIN detection. Solid curve: excess leak as a function of wavelength, measured for Fomalhaut (average and error bars are based on 7 null sequences, recorded on July 16 \& 17, 2008). Shaded grey area: bracketing +/$1 \sigma$ confidence interval (Mennesson et al. in prep). 


\subsection{Overall results (work in progress)}

We have started to search for general trends in the overall sample of 40 single main sequence stars $(8$ spectroscopic binaries left out for now) observed with the KIN, looking for possible correlations between the observed excess and stellar characteristics.

Figure 7 shows the number of excess detected by KIN as a function spectral type, illustrating the fact that excesses are found more frequently around A stars, although statistics are quite limited by the sample size. As A stars are in average younger than later type stars, this could also be an age effect.

\section{\# of Stars}

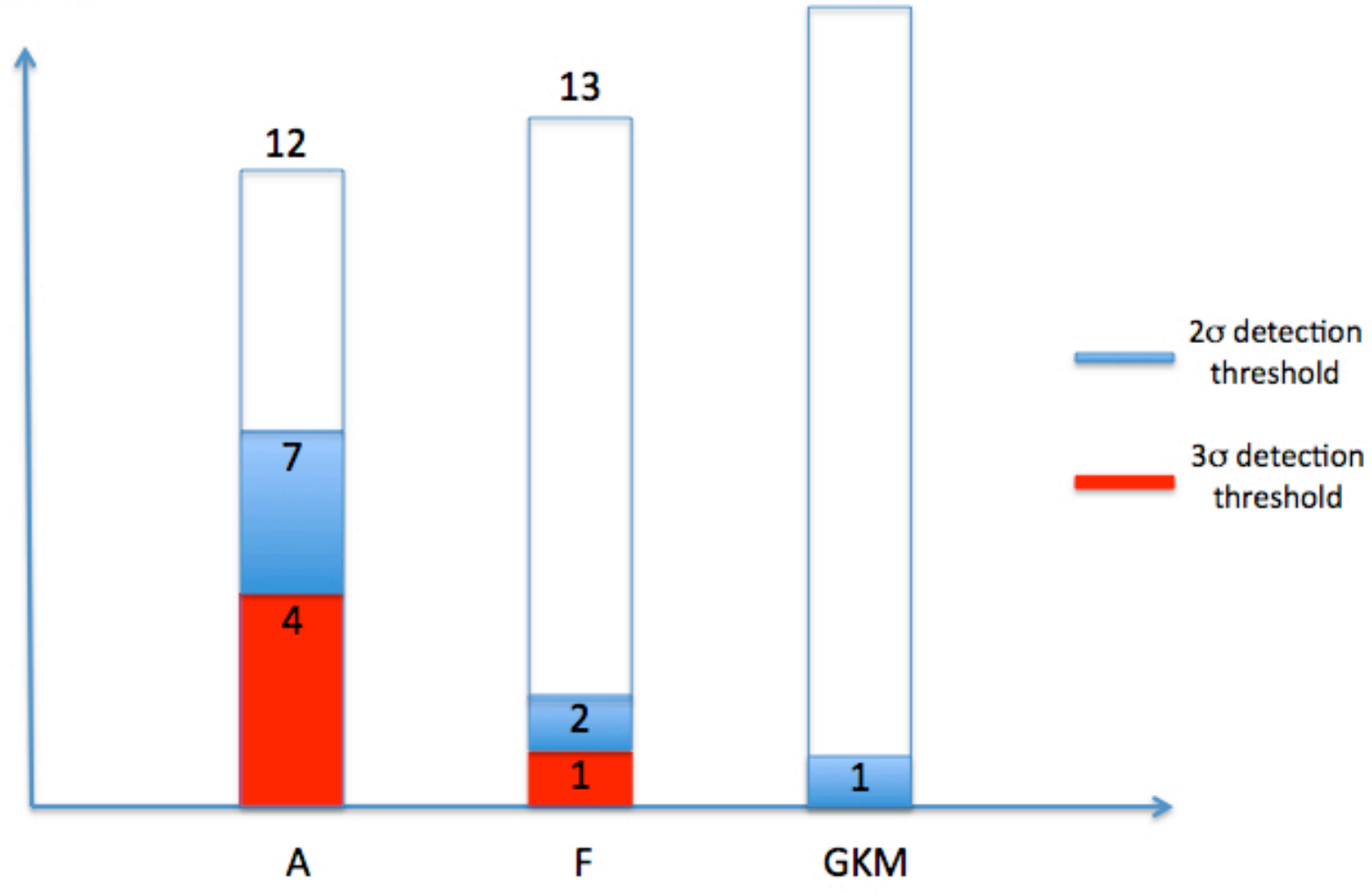

Figure 7: KIN measured excess rate vs spectral type. Depending on the detection threshold used, 4 to 7 A-type stars (out of 12 observed) show a KIN excess (averaged between 8 and 11 microns). In comparison, very few excesses are detected around FGKM-type stars.

Figure 8 shows the average excess leak vs wavelength measured by the KIN for stars of different spectral types, once again stressing the distinct signature of A and B stars versus later spectral types. In any given category, we computed the mean of the excesses observed for all stars falling in that category (with equal weighting), and computed the statistical error bar on the mean. 

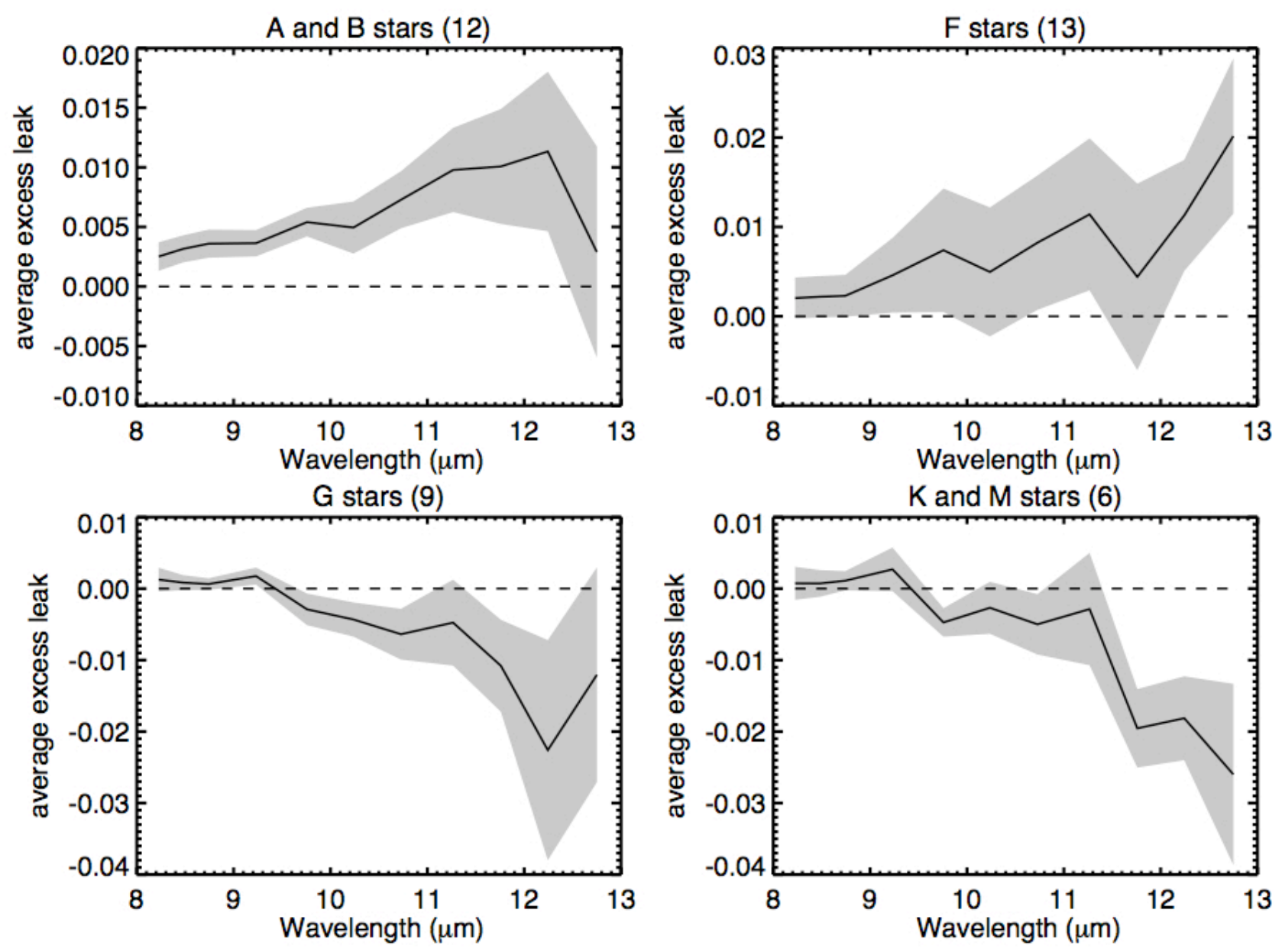

Figure 8: Average excess leak measured by the KIN for stars of different spectral types. The number of stars present in each category is indicated between parentheses.

Similarly, figure 9 shows the average excess leak vs wavelength measured by the KIN for stars belonging to 4 different categories i) stars with known hot excess, ii) stars with known cold excess, iii) stars with known hot and clod excesses, iv) stars with no previously known infrared excesses. The "footprint" of a star with a previously known hot excess is quite different from that of a star with a known cold excess, or that of a star with no known excess. 

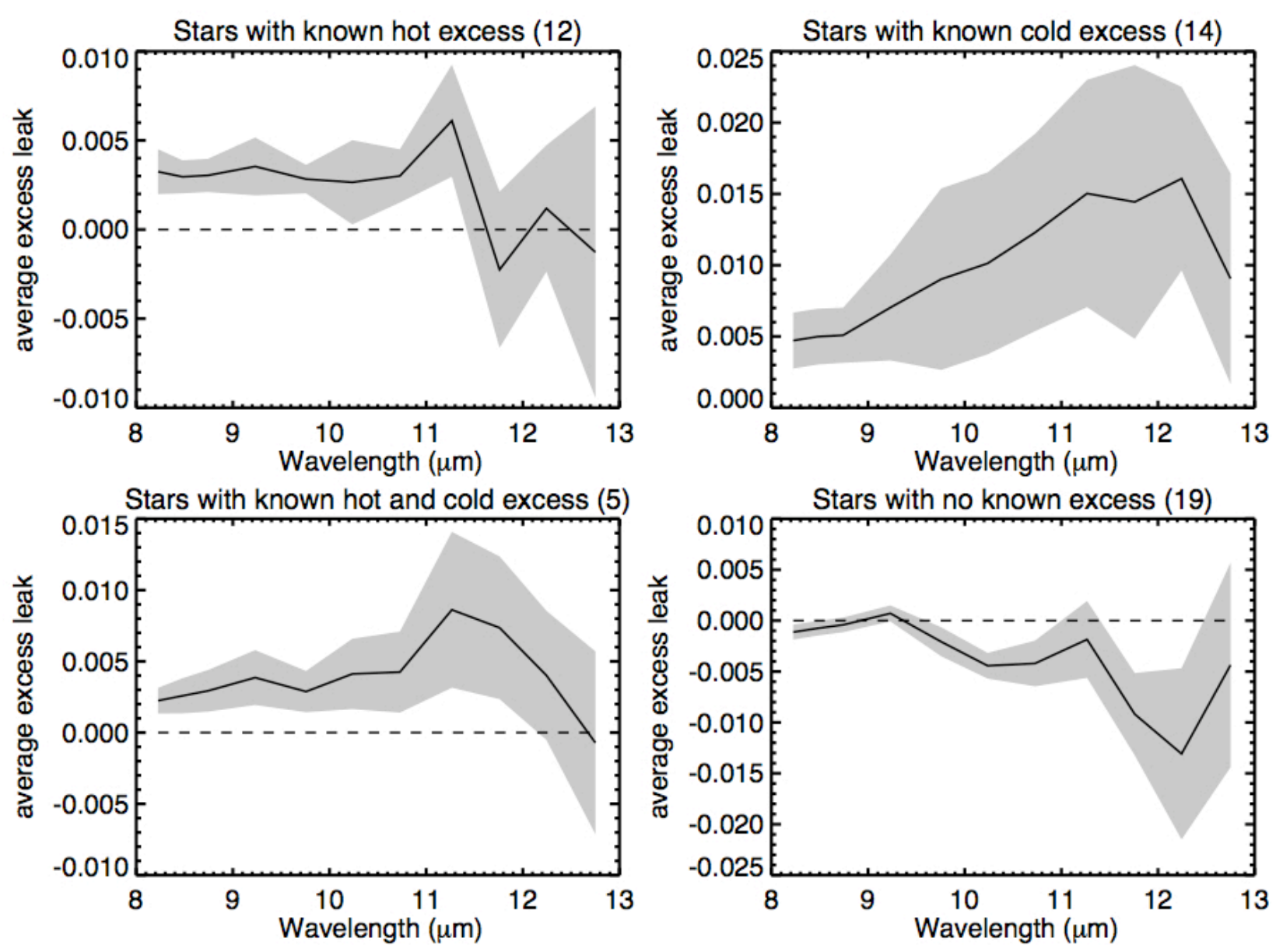

Figure 9: Average excess leak measured by the KIN for stars with different types of dust previously evidenced (or not). The number of stars present in each category is indicated between parentheses.

\section{REFERENCES}

[1] Serabyn, E., Mennesson, B., Colavita, M.M., Koresko, C. and Kuchner, M.J., "The Keck Interferometer Nuller", ApJ, 748, 55 (2012)

[2] Colavita, M.M. et al., "Keck Interferometer Nuller Data reduction and On-Sky Performance", PASP, 121, 1120 (2009)

[3] Sloan, G. C., Price, S. D. "The Infrared Spectral Classification of Oxygen-rich Dust Shells", ApJS, 119,141 (1998)

[4] Mennesson, B et al, "The Dusty AGB Star RS CrB: First Mid-Infrared Interferometric Observations with the Keck Telescopes”, ApJ, 634, 169 (2005)

[5] Ohnaka, et al., "Mid-infrared Interferometry of the Mira variable RR Sco with the VLTI MIDI instrument", A\&A, 429, 1057 (2005)

[6] Barry, R. et al. "Milliarcsecond N-Band Observations of the Nova RS Ophiuchi: First Science with the Keck Interferometer Nuller", ApJ 677, 1253 (2008)

[7] Stark, C. et al. "51 Ophiuchus: A Possible Beta Pictoris Analog Measured with the Keck Interferometer Nuller", ApJ 703, 1188 (2009) 
[8] Ragland S. et al. "First Keck Nulling Observations of a Young Stellar Object: Probing the Circumstellar Environment of the Herbig Ae Star MWC 325", ApJ 746, 126 (2012)

[9] Stark, C. and Cahoy, K., "Stacking the Hay: Modeling the Worst-Case Scenario for Exozodiacal Clouds", AAS Meeting \#218, \#218.07; Bulletin of the American Astronomical Society, Vol. 43 (2011)

[10] Beichman, C. and Velusamy, T., "Effects of Confusion By Zodiacal Light Structures on The Detection of Terrestrial Planets", American Astronomical Society, 191st AAS Meeting, \#64.03; Bulletin of the American Astronomical Society, Vol. 29, p.1310 (1997)

[11] Mennesson, B. and Mariotti J.M. "Array Configurations for a Space Infrared Nulling Interferometer Dedicated to the Search for Earthlike Extrasolar Planets", Icarus, 128, 202 (1997)

[12] Defrère, D. et al. "Nulling interferometry: impact of exozodiacal clouds on the performance of future lifefinding space missions", A\&A, 509, 9 (2010)

[13] Millan-Gabet R. et al., "Exo-Zodiacal Dust Levels for Nearby Main-Sequence Stars: Survey with the Keck Interferometer Nuller" (2011) 